\title{
DISPERSÃO, DISTRIBUIÇÃO ESPACIAL E ESTRATIFICAÇÃO VERTICAL DA COMUNIDADE ARBÓREA EM UM FRAGMENTO FLORESTAL NO PLANALTO CATARINENSE ${ }^{1}$
}

\author{
Marcelo Negrini ${ }^{2}$, Manoela Drews de Aguiar ${ }^{2}$, Cenir Teodoro Vieira ${ }^{3}$, Ana Carolina da Silva ${ }^{4}$ e Pedro \\ Higuchi $^{4}$
}

\begin{abstract}
RESUMO - Este estudo teve como objetivo identificar as estratégias de dispersão de propágulos, a distribuição espacial e a estratificação vertical de espécies arbóreas em um fragmento de Floresta Ombrófila Mista em Lages, SC. Para amostragem da vegetação arbórea, foram alocadas 25 parcelas de $400 \mathrm{~m}^{2}(20 \mathrm{~m} \times 20 \mathrm{~m})$ e todos os indivíduos arbóreos dentro das parcelas com diâmetro a altura do peito (DAP, medido a 1,30 m do solo) maior ou igual a $5 \mathrm{~cm}$ foram identificados e tiveram sua altura estimada. Para complementar a lista florística, foram feitos caminhamentos aleatórios no fragmento objetivando identificar espécies arbóreas não amostradas nas parcelas. As espécies foram classificadas segundo: i) a sua síndrome de dispersão em zoocórica, anemocórica ou autocórica; ii) o padrão de distribuição espacial dos indivíduos, considerando-se a distribuição aleatória, agregada e uniforme; iii) a posição no estrato vertical da floresta, como sendo dos estratos superior, intermediário ou inferior. Do total de 87 espécies amostradas, $80,5 \%$ foram classificadas como zoocóricas, 16,1\% como anemocóricas e 3,4\% como autocóricas. Verificou-se a predominância de espécies com distribuição espacial aleatória e pertencente ao estrato superior, com altura superior ou igual a 12,45 m. Os resultados indicam a importância da fauna silvestre para a manutenção do funcionamento ecológico do fragmento estudado, uma vez que a maior parte das espécies são zoocóricas. O conhecimento desses atributos das populações arbóreas pode subsidiar estratégias de conservação e manejo de fragmentos florestais na região, uma vez que permite conhecer melhor a ecologia das espécies.
\end{abstract}

Palavras-chave: Floresta Ombrófila Mista, altura de árvores e síndromes de dispersão.

\section{DISPERSION, SPATIAL DISTRIBUTION AND VERTICAL STRATIFICATION OF THE TREE COMMUNITY IN A FOREST FRAGMENT IN "PLANALTO CATARINENSE" REGION}

\begin{abstract}
This study aimed to identify the strategies of propagules dispersion, spatial distribution and vertical stratification of tree species in an Araucaria Forest in Lages, SC. For sampling of the tree component, 25, $20 \times 20 \mathrm{~m}(400 \mathrm{~m} 2)$, plots were allocated, where all living trees with diameter at breast height (dbh, measured at 1,30 $\mathrm{m}$ height) higher or equal to $5 \mathrm{~cm}$ had the botanical identity determined and the total height estimated. Tree species found outside the plots, through random walks in the fragment, were recorded in order to complement the floristic list. The species were classified according to: $i)$ their dispersion syndrome in zoochory, anemochory and autochory; ii) spatial distribution patterns of individuals, considering the random, clustered and regular distributions; and iii) vertical position in forest, being categorized as upper, intermediary
\end{abstract}

\footnotetext{
${ }^{1}$ Recebido em 01.04.2011 aceito para publicação em 04.06.2012.

${ }^{2}$ Engenheiros florestais - Universidade do Estado de Santa Catarina, UDESC, Brasil. E-mail: <engfmarcelonegrini@ hotmail.com> e $<$ manoo_aguiar@hotmail.com>.

${ }^{3}$ Ministério Público do Estado do Mato Grosso do Sul, Corpo Técnico. E-mail: <cenir_vieira@mp.ms.gov.br>.

${ }^{4}$ Universidade do Estado de Santa Catarina (UDESC), Centro de Ciências Agroveterinárias, Departamento de Engenharia Florestal. E-mail: <carol_sil4@yahoo.com.br>e <higuchip@gmail.com>
} 
and lower stratum. The survey totaled 87 species, with the predominance of zoochoric, random distributed species, occupying the upper vertical stratum. The knowledge of these attributes of tree species populations may support the conservation and management strategies offorest fragments in the study region, since it allow a better understanding of species ecology.

Keywords: Araucaria Forest, Tree height and Dispersion syndrome.

\section{INTRODUÇÃO}

Em ecossistemas florestais, o conhecimento sobre alguns aspectos ecológicos, como as síndromes de dispersão de propágulos, a distribuição espacial dos indivíduos e a partição do perfil vertical da floresta pelas espécies, é fundamental para a tomada de decisões em planos de conservação e manejo. Diversos modelos foram propostos na tentativa de explicar o funcionamento da dispersão de propágulos. De acordo com o modelo proposto por Janzen (1970) e Connell (1971), as espécies arbóreas desenvolveram várias estratégias adaptativas relacionadas à maior capacidade de dispersão de propágulos em resposta à pressão seletiva associada à maior taxa de mortalidade que ocorre próxima à árvore matriz. Quanto mais próximo da planta-mãe, maior seria a densidade de indivíduos coespecíficos, o que resultaria em maior competição intraespecífica e maior atração de predadores específicos de sementes ou plântulas (mortalidade dependente da densidade ou teoria da fuga). Entretanto, de acordo com Hubbell (1980), como a chuva de sementes é mais intensa próximo da plantamãe, isso compensaria os fatores de mortalidade dependentes de densidade, promovendo recrutamento maior ao redor da planta-mãe. Já Augspurger (1984) propôs a teoria de colonização, em que a dispersão à maior distância aumentaria a probabilidade de as progênies encontrarem um hábitat adequado, aumentando, assim, a sua sobrevivência. Entretanto, não há um único padrão para todas as espécies. Em alguns estudos, o modelo de Janzen (1970) e Connell (1971) foi o que teve maior aplicabilidade (PERES et al., 1997; WRIGHT; DUBER, 2001) e em outros não (BLATE et al., 1998; ZIPARRO; MORELLATO, 2005). Isso acontece porque há outros fatores ecológicos, além da mortalidade dependente da densidade, como a capacidade de dispersão de propágulos e a existência de filtros ambientais, que podem influenciar no padrão de distribuição espacial dos propágulos.

A dispersão de propágulos, além de outros fatores, pode influenciar na distribuição espacial dos indivíduos de determinada espécie de árvore, que pode ser agregada, aleatória ou uniforme. No caso de dispersão por animais (zoocoria), a distribuição espacial pode assumir diferentes arranjos, dependendo do tipo de dispersor. Na zoocoria, como na dispersão por aves, existe uma tendência à formação de agregados, uma vez que as sementes são dispersas em locais onde os animais permanecem, como ninhos, poleiros etc. (URBANETZ et al., 2003). Segundo esses mesmos autores, no caso de uma dispersão secundária poderia ser obtido um padrão espacial uniforme, a exemplo de quando os agentes dispersores são as formigas, pois estas geralmente possuem ninhos uniformemente distribuídos por uma área. Os frutos leves e, ou, que possuem estruturas de flutuação são dispersos pelo vento (anemocoria), o que normalmente resulta na dispersão aleatória, enquanto o fruto pesado e seco, cuja dispersão é pela gravidade (barocoria), pode ocasionar distribuição espacial agregada.

O estrato que uma árvore ocupa na floresta pode ter estreita relação com a síndrome de dispersão de propágulos. Em estudo realizado por Giehl et al. (2007), foi constatado que muitos indivíduos de espécies anemocóricas estão em classes superiores de altura, o que facilita a ação dos ventos na dispersão de propágulos. Já nas espécies zoocóricas não seria necessário porte mais elevado. No estudo de Leyser et al. (2009), por exemplo, foi identificado que espécies zoocóricas estariam divididas por todas as classes de altura.

Considerando que os fragmentos florestais são elementos importantes na paisagem, pois desempenham relevante função ambiental (e.g. proteção do solo e cursos de água, sequestro de carbono, hábitat para a fauna e fonte de propágulos, entre outras) e apresentam elevada biodiversidade, estudos que buscam entender a ecologia dessas áreas são relevantes. Estudos sobre a florística e, ou, a estrutura de remanescentes florestais têm sido realizados no Planalto Catarinense (e. g. FALKENBERG, 2003; FORMENTO et al., 2004; ESKUCHE, 2007; HIGUCHI et al., 2012; SILVA et al., 2012; HIGUCHI et al., no prelo), no entanto pouco

Revista Árvore, Viçosa-MG, v.36, n.5, p.919-929, 2012 
se conhece sobre aspectos quantitativos relacionados às síndromes de dispersão, distribuição espacial e estratificação vertical da comunidade arbórea. Dessa forma, os objetivos deste trabalho foram identificar as estratégias de dispersão de propágulos, a distribuição espacial e a estratificação vertical de espécies arbóreas em um fragmento de Floresta Ombrófila Mista em Lages, SC, como subsídio para definição de estratégias de manejo e conservação.

\section{MATERIAL E MÉTODOS}

O estudo foi realizado em fragmento de Floresta Ombrófila Mista Montana (IBGE, 1992), com área total de 22 ha, localizado em Lages, região do Planalto Catarinense. Esse fragmento passou por cortes seletivos no passado, encontrando-se em estágio avançado de recuperação pós-distúrbio. O município está localizado na latitude $27^{\circ} 48^{\prime} 58^{\prime \prime}$ Sul e longitude $50^{\circ} 19^{\prime} 30^{\prime \prime}$ Oeste, com altitude em torno de $916 \mathrm{~m}$. O clima predominante na região é $\mathrm{Cfb}$, de acordo com a classificação de Köppen, sendo a precipitação anual média de $1.479,48$ mm, com chuvas bem distribuídas durante o ano. A temperatura anual média é de $16^{\circ} \mathrm{C}$ (BRASIL, 1992).

Para amostragem da vegetação arbórea, foram alocadas, no estudo de Silva et al. (2012), 25 parcelas de $400 \mathrm{~m}^{2}(20 \times 20 \mathrm{~m})$ distribuídas na área de forma estratificada-sistemática, de modo a amostrar as variações ambientais presentes. Todos os indivíduos arbóreos com diâmetro à altura do peito (DAP, medido a $1,30 \mathrm{~m}$ do solo) superior ou igual a $5 \mathrm{~cm}$, situados dentro das parcelas, foram identificados e tiveram sua altura estimada com o auxílio de um podão com altura conhecida. Para complementar a lista florística de espécies, foram feitos caminhamentos aleatórios no fragmento, a fim de identificar espécies arbóreas não amostradas nas parcelas. As espécies tiveram sua abundância quantificada (número de indivíduos) e foram classificadas em famílias de acordo com o sistema APG III (ANGIOSPERM PHYLOGENY GROUP, 2009).

A síndrome de dispersão de propágulos de cada espécie encontrada no fragmento foi definida de acordo com a metodologia de Van der Pijl (1982), em que: i) espécies de frutos carnosos e com outros elementos que evidenciam a dispersão por animais foram consideradas como de dispersão zoocórica; ii) frutos ou sementes com alas e mecanismos para flutuação foram definidos como de dispersão anemocórica; e iii) frutos tipo cápsula deiscente, que, ao se abrir, libera as sementes com um rápido movimento, foram considerados de dispersão autocórica. A determinação dessas síndromes deu-se por observações dos diásporos e por consultas em literatura (LORENZI, 1998a; LORENZI, 1998b; RONDON NETO et al., 2001; BUDKE et al., 2005; GIEHL et al., 2007; ALMEIDA et al., 2008; LEYSER et al., 2009; LORENZI, 2009).

Com o objetivo de verificar a distribuição espacial das espécies na área, foi calculado, para cada população das espécies amostradas nas parcelas (população com no mínimo dois indivíduos), o índice de Morisita (MORISITA, 1962) modificado por Smith-Gill (1975): Índice de Morisita Padronizado. Neste índice, se o valor padronizado é maior que 0,5 , a distribuição espacial da população é agregada; se é menor que -0,5, a distribuição é uniforme; se está entre esses dois valores, é aleatória.

O perfil vertical da floresta foi estratificado conforme metodologia citada por Souza et al. (2003): $\mathrm{H} 1<(\mathrm{h}-\mathrm{dp}),(\mathrm{h}-\mathrm{dp}) \leq \mathrm{H} 2<(\mathrm{h}+\mathrm{dp}), \mathrm{H} 3 \geq(\mathrm{h}$ $+\mathrm{dp}$ ), em que Hi representa as alturas-limite para os estratos inferior, intermediário e superior; h representa a altura média de todos os indivíduos amostrados e dp o desvio-padrão das alturas. Após a definição dos valores-limite, as espécies foram agrupadas nas classes: inferior, espécies menores que 4,74 m; intermediário, espécies com altura superior ou igual a 4,74 m e menor que $12,45 \mathrm{~m}$; e superior, espécies com valores superiores ou iguais a $12,45 \mathrm{~m}$. Para essa classificação, considerou-se o porte das espécies em sua fase adulta, tendo como base as alturas observadas neste estudo, no estudo de Higuchi et al. (2012) e em observações pessoais.

\section{RESULTADOS}

No fragmento estudado foram encontradas 87 espécies arbóreas, distribuídas em 36 famílias, sendo a família Myrtaceae a de maior riqueza, com 18 espécies (20,7\% do total) (Tabela 1). Todas as espécies de Myrtaceae são zoocóricas e, considerando as espécies de todas as famílias encontradas na área, 80,5\% apresentaram zoocoria como síndrome de dispersão, $16,1 \%$ anemocoria e apenas $3,4 \%$ autocoria (Figura 1A). A autocoria ocorreu em apenas três espécies, todas da família Euphorbiaceae: Sebastiania commersoniana (Baill.) L.B. Sm. \& Downs, Sapium glandulosum (L.) Morong e Sebastiania brasiliensis Spreng.

Revista Árvore, Viçosa-MG, v.36, n.5, p.919-929, 2012 
Tabela 1 - Espécies encontradas em um fragmento de Floresta Ombrófila Mista Montana em Lages, SC, com a respectiva estratégia de dispersão (D), valor do Índice de Morisita Padronizado (IMP), distribuição espacial (DE) e estratificação vertical (EV). Em que: Z - zoocórica; An - anemocórica; Au - autocórica; Ag - agregado; Al - aleatório; S - estrato superior; Int - estrato intermediário; Inf - estrato inferior; * - espécies registradas somente na florística, que não tiveram sua altura estimada nem foram quantificadas; ** - espécies que ocorreram em uma única parcela amostral, que não tiveram calculado o IMP.

Table 1 - Tree species found in an Araucaria Forest fragment in the municipality of Lages, SC, with their respective dispersal strategies $(D)$, values of the Standardized Morisita Index (IMP), spatial distribution (DE) and vertical stratification (VE). Where: Z-zoochoric; An - anemocoric; Au - autochoric; Ag - aggregate; Al - random; S - upper stratum, Int - intermediary stratum; Inf - lower; * species found only in the floristic survey with no height estimative. ** - species occurring in only one sample, with no IMP calculated.

\begin{tabular}{|c|c|c|c|c|}
\hline Táxon & $\mathrm{D}$ & IMP & $\mathrm{DE}$ & EV \\
\hline ANACARDIACEAE & & & & \\
\hline Lithraea brasiliensis Marchand & $\mathrm{Z}$ & 0,5129 & $\mathrm{Ag}$ & $\mathrm{S}$ \\
\hline Schinus polygamus (Cav.) Cabrera & $\mathrm{Z}$ & $*$ & $*$ & $*$ \\
\hline $\begin{array}{l}\text { Schinus terebinthifolius Raddi } \\
\text { ANNONACEAE }\end{array}$ & $\mathrm{Z}$ & 0,4773 & $\mathrm{Al}$ & Int \\
\hline $\begin{array}{l}\text { Annona rugulosa (Schltdl.) H.Rainer } \\
\text { AQUIFOLIACEAE }\end{array}$ & $\mathrm{Z}$ & 0,4122 & $\mathrm{Al}$ & Int \\
\hline Ilex brevicuspis Reissek & $\mathrm{Z}$ & $-0,1293$ & Al & $\mathrm{S}$ \\
\hline Ilex dumosa Reissek & $\mathrm{Z}$ & $* *$ & $* *$ & Int \\
\hline Ilex paraguariensis A.St.-Hil. & $\mathrm{Z}$ & 0,3092 & Al & $\mathrm{S}$ \\
\hline $\begin{array}{l}\text { Ilex theezans Mart. ex Reissek } \\
\text { ARALIACEAE }\end{array}$ & $\mathrm{Z}$ & 0,1085 & $\mathrm{Al}$ & $\mathrm{S}$ \\
\hline $\begin{array}{l}\text { Oreopanax fulvus Marchal } \\
\text { ARAUCARIACEAE }\end{array}$ & $\mathrm{Z}$ & $-0,2586$ & $\mathrm{Al}$ & Int \\
\hline $\begin{array}{l}\text { Araucaria angustifolia (Bert.) Kuntze } \\
\text { ASTERACEAE }\end{array}$ & $\mathrm{Z}$ & 0,5090 & $\mathrm{Ag}$ & $\mathrm{S}$ \\
\hline Baccharis semiserrata DC. & An & $*$ & $*$ & $*$ \\
\hline Baccharis uncinella DC. & An & $*$ & $*$ & $*$ \\
\hline Dasyphyllum spinescens (Less.) Cabrera & An & 0,5555 & $\mathrm{Ag}$ & $\mathrm{S}$ \\
\hline Dasyphyllum tomentosum (Spreng.) Cabrera & An & 0,4142 & $\mathrm{Al}$ & $\mathrm{S}$ \\
\hline Gochnatia polymorpha (Less.) Cabrera & An & 0,1790 & $\mathrm{Al}$ & Int \\
\hline $\begin{array}{l}\text { Vernonanthura discolor (Spreng.) H.Rob. } \\
\text { BERBERIDACEAE }\end{array}$ & An & 0,5023 & $\mathrm{Ag}$ & $\mathrm{S}$ \\
\hline $\begin{array}{l}\text { Berberis laurina Billb. } \\
\text { BIGNONIACEAE }\end{array}$ & $\mathrm{Z}$ & $*$ & $*$ & $*$ \\
\hline Handroanthus albus (Cham.) Mattos & An & 0,6689 & $\mathrm{Ag}$ & Int \\
\hline $\begin{array}{l}\text { Jacaranda puberula Cham. } \\
\text { CANELLACEAE }\end{array}$ & An & 0,5619 & $\mathrm{Ag}$ & $\mathrm{S}$ \\
\hline $\begin{array}{l}\text { Cinnamodendron dinisii Schwacke } \\
\text { CANNABACEAE }\end{array}$ & $\mathrm{Z}$ & 0,5345 & $\mathrm{Ag}$ & S \\
\hline $\begin{array}{l}\text { Celtis brasiliensis (Gardner) Planch. } \\
\text { CELASTRACEAE }\end{array}$ & $\mathrm{Z}$ & 0,5620 & $\mathrm{Ag}$ & Int \\
\hline Maytenus boaria Molina & $\mathrm{Z}$ & $* *$ & $* *$ & Int \\
\hline Maytenus evonymoides Reissek & $\mathrm{Z}$ & 0,5586 & $\mathrm{Ag}$ & $\mathrm{S}$ \\
\hline $\begin{array}{l}\text { Maytenus muelleri Schwacke } \\
\text { CLETHRACEAE }\end{array}$ & $\mathrm{Z}$ & 0,1953 & $\mathrm{Al}$ & Int \\
\hline $\begin{array}{l}\text { Clethra scabra } \text { Pers. } \\
\text { CUNONIACEAE }\end{array}$ & An & 0,3092 & $\mathrm{Al}$ & S \\
\hline $\begin{array}{l}\text { Lamanonia ternata Vell. } \\
\text { DICKSONIACEAE }\end{array}$ & An & $* *$ & $* *$ & S \\
\hline Dicksonia sellowiana Hook. & An & 0,6912 & $\mathrm{Ag}$ & Int \\
\hline
\end{tabular}

Revista Árvore, Viçosa-MG, v.36, n.5, p.919-929, 2012 
Dispersão, distribuição espacial e estratificação vertical...

Tabela 1 - Cont

Table 1 - Cont

\begin{tabular}{|c|c|c|c|c|}
\hline Táxon & $\mathrm{D}$ & IMP & $\mathrm{DE}$ & EV \\
\hline \multicolumn{5}{|l|}{ ERYTHROXYLACEAE } \\
\hline Erythroxylum cuneifolium (Mart.) O.E. Schulz & $\mathrm{Z}$ & 0,6257 & $\mathrm{Ag}$ & Int \\
\hline $\begin{array}{l}\text { Erythroxylum deciduum A.St.-Hil. } \\
\text { EUPHORBIACEAE }\end{array}$ & $\mathrm{Z}$ & 0,3092 & $\mathrm{Al}$ & Int \\
\hline Sapium glandulosum (L.) Morong & $\mathrm{Au}$ & $-0,0431$ & $\mathrm{Al}$ & $\mathrm{S}$ \\
\hline Sebastiania brasiliensis Spreng. & $\mathrm{Au}$ & 0,9362 & $\mathrm{Ag}$ & In \\
\hline $\begin{array}{l}\text { Sebastiania commersoniana (Baill.) L. B. Sm. \& Downs } \\
\text { FABACEAE }\end{array}$ & $\mathrm{Au}$ & 0,6685 & $\mathrm{Ag}$ & $\mathrm{S}$ \\
\hline $\begin{array}{l}\text { Dalbergia frutescens (Vell.) Britton } \\
\text { LAURACEAE }\end{array}$ & An & 0,4628 & $\mathrm{Al}$ & $\mathrm{S}$ \\
\hline Nectandra lanceolata Nees & $\mathrm{Z}$ & 0,3092 & $\mathrm{Al}$ & $\mathrm{S}$ \\
\hline Nectandra megapotamica (Spreng.) Mez & $\mathrm{Z}$ & 0,5376 & $\mathrm{Ag}$ & $\mathrm{S}$ \\
\hline Ocotea puberula (Rich.) Nees & $\mathrm{Z}$ & 0,1953 & $\mathrm{Al}$ & $\mathrm{S}$ \\
\hline Ocotea pulchella Mart. & $\mathrm{Z}$ & 0,2170 & $\mathrm{Al}$ & $\mathrm{S}$ \\
\hline $\begin{array}{l}\text { Persea major L.E. Kopp } \\
\text { LOGANIACEAE }\end{array}$ & $\mathrm{Z}$ & $-0,0431$ & $\mathrm{Al}$ & $\mathrm{S}$ \\
\hline $\begin{array}{l}\text { Strychnos brasiliensis (Spreng.) Mart. } \\
\text { MELASTOMATACEAE }\end{array}$ & $\mathrm{Z}$ & $-0,1293$ & $\mathrm{Al}$ & Inf \\
\hline Leandra regnellii (Triana) Cogn. & $\mathrm{Z}$ & $*$ & $*$ & * \\
\hline Miconia cinerascens Miq. & $\mathrm{Z}$ & $-0,0862$ & $\mathrm{Al}$ & In \\
\hline $\begin{array}{l}\text { Miconia hiemalis A.St.-Hil. \& Naudin ex Naudin } \\
\text { MYRTACEAE }\end{array}$ & $\mathrm{Z}$ & $*$ & $*$ & $*$ \\
\hline Acca sellowiana (O.Berg) Burret & $\mathrm{Z}$ & 0,5086 & $\mathrm{Ag}$ & Inf \\
\hline Blepharocalyx salicifolius (Kunth) O. Berg & $\mathrm{Z}$ & 0,1085 & $\mathrm{Al}$ & Int \\
\hline Calyptranthes concinna DC. & $\mathrm{Z}$ & 0,2820 & $\mathrm{Al}$ & In \\
\hline Campomanesia xanthocarpa $\mathrm{O}$. Berg & $\mathrm{Z}$ & 0,5091 & $\mathrm{Ag}$ & $\mathrm{S}$ \\
\hline Eugenia pluriflora DC. & $\mathrm{Z}$ & 0,1085 & $\mathrm{Al}$ & In \\
\hline Eugenia pyriformis Cambess. & $\mathrm{Z}$ & $-0,1054$ & $\mathrm{Al}$ & $\mathrm{S}$ \\
\hline Eugenia sp. & $\mathrm{Z}$ & 0,3797 & $\mathrm{~A} 1$ & Int \\
\hline Eugenia uruguayensis Cambess. & $\mathrm{Z}$ & 0,4773 & $\mathrm{Al}$ & In \\
\hline Myrceugenia euosma (O. Berg) D. Legrand & $\mathrm{Z}$ & $* *$ & $* *$ & Int \\
\hline Myrceugenia oxysepala (Burret) D. Legrand \& Kausel & $\mathrm{Z}$ & 0,4773 & $\mathrm{Al}$ & In \\
\hline Myrcia bombycina $(\mathrm{O}$. Berg) Nied. & $\mathrm{Z}$ & 0,6457 & $\mathrm{Ag}$ & In \\
\hline Myrcia guianensis (Aubl.) DC. & $\mathrm{Z}$ & $-0,0732$ & $\mathrm{Al}$ & $\mathrm{S}$ \\
\hline Myrcia hatschbachii D. Legrand & $\mathrm{Z}$ & $-0,1724$ & $\mathrm{Al}$ & $\mathrm{S}$ \\
\hline Myrcia laruotteana Cambess. & $\mathrm{Z}$ & 0,1953 & $\mathrm{~A} 1$ & Int \\
\hline Myrcia palustris DC. & $\mathrm{Z}$ & 0,4142 & $\mathrm{Al}$ & In \\
\hline Myrcia splendens ( $\mathrm{Sw}$.$) DC.$ & $\mathrm{Z}$ & 0,5186 & $\mathrm{Ag}$ & $\mathrm{S}$ \\
\hline Myrciaria delicatula (DC.) O. Berg & $\mathrm{Z}$ & $-0,0431$ & $\mathrm{Al}$ & In \\
\hline $\begin{array}{l}\text { Myrrhinium atropurpureum Schott } \\
\text { PODOCARPACEAE }\end{array}$ & $\mathrm{Z}$ & 0,5293 & $\mathrm{Ag}$ & In \\
\hline $\begin{array}{l}\text { Podocarpus lambertii Klotzsch } \\
\text { PRIMULACEAE }\end{array}$ & $\mathrm{Z}$ & 0,5038 & $\mathrm{Ag}$ & $\mathrm{S}$ \\
\hline Myrsine coriacea (Sw.) Roem. \& Schult. & $\mathrm{Z}$ & $-0,1724$ & $\mathrm{Al}$ & $\mathrm{S}$ \\
\hline Myrsine gardneriana A. DC. & $\mathrm{Z}$ & $-0,0431$ & $\mathrm{~A} 1$ & $\mathrm{~S}$ \\
\hline $\begin{array}{l}\text { Myrsine umbellata Mart. } \\
\text { PROTEACEAE }\end{array}$ & $\mathrm{Z}$ & 0,5007 & $\mathrm{Ag}$ & $\mathrm{S}$ \\
\hline $\begin{array}{l}\text { Roupala montana Aubl. } \\
\text { QUILLAJACEAE }\end{array}$ & An & 0,4122 & $\mathrm{Al}$ & $\mathrm{S}$ \\
\hline Quillaja brasiliensis (A.St.-Hil. \& Tul.) Mart. & An & $* *$ & $* *$ & In \\
\hline
\end{tabular}


Tabela 1 - Cont.

Table 1 - Cont.

\begin{tabular}{|c|c|c|c|c|}
\hline Táxon & $\mathrm{D}$ & IMP & DE & EV \\
\hline \multicolumn{5}{|l|}{ RHAMNACEAE } \\
\hline Scutia buxifolia Reissek & $\mathrm{Z}$ & 0,5239 & $\mathrm{Ag}$ & $\mathrm{S}$ \\
\hline ROSACEAE & & & & \\
\hline $\begin{array}{l}\text { Prunus myrtifolia (L.) Urb. } \\
\text { RUTACEAE }\end{array}$ & $\mathrm{Z}$ & 0,5835 & $\mathrm{Ag}$ & S \\
\hline Zanthoxylum kleinii (R.S.Cowan) P.G. Waterman & $\mathrm{Z}$ & 0,5309 & $\mathrm{Ag}$ & S \\
\hline $\begin{array}{l}\text { Zanthoxylum rhoifolium Lam. } \\
\text { SALICACEAE }\end{array}$ & \multicolumn{3}{|c|}{ SALICACEAE } & S \\
\hline Banara tomentosa $\mathrm{Clos}$ & $\mathrm{Z}$ & 0,5125 & $\mathrm{Ag}$ & Int \\
\hline Casearia decandra Jacq. & $\mathrm{Z}$ & 0,5024 & $\mathrm{Ag}$ & $\mathrm{S}$ \\
\hline Casearia obliqua Spreng. & $\mathrm{Z}$ & 0,5763 & $\mathrm{Ag}$ & S \\
\hline Xylosma ciliatifolia (Clos) Eichler & $\mathrm{Z}$ & 0,3580 & $\mathrm{Al}$ & $\mathrm{S}$ \\
\hline $\begin{array}{l}\text { Xylosma tweediana (Clos) Eichler } \\
\text { SAPINDACEAE }\end{array}$ & $\mathrm{Z}$ & $-0,0862$ & $\mathrm{Al}$ & Int \\
\hline Allophylus guaraniticus (A.St.-Hil.) Radlk & $\mathrm{Z}$ & 0,3232 & $\mathrm{~A} 1$ & Int \\
\hline Cupania vernalis Cambess. & $\mathrm{Z}$ & 0,5365 & $\mathrm{Ag}$ & $\mathrm{S}$ \\
\hline Matayba elaeagnoides Radlk & $\mathrm{Z}$ & 0,5236 & $\mathrm{Ag}$ & S \\
\hline \multicolumn{5}{|l|}{ SOLANACEAE } \\
\hline Brunfelsia cuneifolia J.A. Shimidt & $\mathrm{Z}$ & $*$ & $*$ & $*$ \\
\hline Solanum pabstii L.B. Sm. \& Downs & $\mathrm{Z}$ & 0,1790 & $\mathrm{Al}$ & Int \\
\hline $\begin{array}{l}\text { Solanum sanctaecatharinae Dunal } \\
\text { STYRACACEAE }\end{array}$ & $\mathrm{Z}$ & 0,2353 & $\mathrm{Al}$ & $\mathrm{S}$ \\
\hline Styrax acuminatus Pohl & $\mathrm{Z}$ & 0,3092 & $\mathrm{Al}$ & Int \\
\hline $\begin{array}{l}\text { Styrax leprosus Hook. \& Arn. } \\
\text { SYMPLOCACEAE }\end{array}$ & $\mathrm{Z}$ & 0,5265 & $\mathrm{Ag}$ & $\mathrm{S}$ \\
\hline $\begin{array}{l}\text { Symplocos uniflora (Pohl) Benth. } \\
\text { THYMELAEACEAE }\end{array}$ & $\mathrm{Z}$ & 0,3580 & $\mathrm{Al}$ & $\mathrm{S}$ \\
\hline $\begin{array}{l}\text { Daphnopsis racemosa Griseb. } \\
\text { VERBENACEAE }\end{array}$ & $\mathrm{Z}$ & $* *$ & $* *$ & Int \\
\hline $\begin{array}{l}\text { Duranta vestita Cham. } \\
\text { WINTERACEAE }\end{array}$ & $\mathrm{Z}$ & 0,5397 & $\mathrm{Ag}$ & Int \\
\hline Drimys brasiliensis Miers & $\mathrm{Z}$ & 0,4856 & $\mathrm{Al}$ & Int \\
\hline
\end{tabular}

Houve predominância de populações com os indivíduos com distribuição espacial aleatória na área estudada (Figura 1B). Para as síndromes de dispersões zoocórica e autocórica, foram observadas predominantemente espécies com a distribuição espacial aleatória e agregada, respectivamente, enquanto o número de espécies anemocóricas com a distribuição espacial agregada e aleatória foi o mesmo (Figura 1C). Entre as três únicas espécies autocóricas, as duas do gênero Sebastiana apresentaram distribuição agregada e Sapium glandulosum aleatória. No entanto, Sapium glandulosum foi representado por somente dois indivíduos na área amostrada, não sendo possível fazer afirmações seguras sobre sua distribuição.
A estratificação vertical da floresta demonstrou predominância de espécies classificadas como do estrato superior, ou seja, com alturas iguais ou superiores a $12,45 \mathrm{~m}$, seguidas por espécies do estrato intermediário, com alturas menores que 12,45 e superiores ou iguais a 4,74 m (Figura 1D). Entre as espécies pertencentes ao dossel superior, destacase como mais abundante Araucaria angustifolia (Bert.) Kuntze, espécie característica da Floresta Ombrófila Mista. Também se destacaram como pertencentes ao estrato superior, com grande número de indivíduos, as espécies: Myrcia splendens (Sw.) DC., Podocarpus lambertii Klotzsch, Jacaranda puberula Cham. e Zanthoxylum kleinii (R.S.Cowan) P.G. Waterman. No estrato intermediário, foram

Revista Árvore, Viçosa-MG, v.36, n.5, p.919-929, 2012 



(C)


Figura 1 - Síndromes de dispersão das espécies (A), distribuição espacial das espécies (B), porcentagem de espécies em cada distribuição espacial e estratégia de dispersão (C), distribuição percentual das espécies em classes de altura, em m (D), e porcentagem de espécies de cada estrato da floresta por estratégia de dispersão (E) em um fragmento de Floresta Ombrófila Mista Montana em Lages, SC.

Figure 1 - Tree species found in an Araucaria Forest fragment in the municipality of Lages, SC, with their respective dispersal strategies (D), values of the Standardized Morisita Index (IMP), spatial distribution (DE) and vertical stratification (VE). Where: Z- zoochoric; An - anemocoric; Au - autochoric; Ag - aggregate; Al - random; $S$ - upper stratum, Int - intermediary stratum; Inf-lower; * species found only in the floristic survey with no height estimative. ** - species occurring in only one sample, with no IMP calculated

encontrados em maior abundância Myrcia bombycina (O. Berg) Nied., Banara tomentosa Clos, Duranta vestita Cham., Dicksonia sellowiana Hook. e Allophylus guaraniticus (A.St.-Hil.) Radlk. As espécies pertencentes ao estrato inferior, que obtiveram altura igual ou inferior a 4,74 m, foram apenas duas: Acca sellowiana (O. Berg) Burret e Strychnos brasiliensis (Spreng.) Mart.

Revista Árvore, Viçosa-MG, v.36, n.5, p.919-929, 2012 
Considerando a síndrome de dispersão de acordo com a estratificação da vegetação, foi observado que a zoocoria ocorre com maior frequência em espécies pertencentes ao estrato superior, seguido de espécies pertencentes ao estrato intermediário (Figura 1E). As únicas duas espécies do estrato inferior também são zoocóricas. Do total de espécies, 10,0\% são anemocóricas pertencentes ao estrato superior e somente 5,0\% são anemocóricas do estrato intermediário. Das três espécies autocóricas, Sebastiania brasiliensis é a única pertencente ao estrato intermediário, sendo as outras integrantes do estrato superior.

\section{DISCUSSÃO}

A síndrome de dispersão zoocórica tem sido relatada como predominante em diferentes fitofisionomias vegetacionais, tanto em ambientes tropicais, a exemplo do Cerrado (GOTTSBERGER; SILBERBAUERGOTTSBERGER, 1983) e da Floresta Estacional Semidecidual (NUNES et al., 2003), quanto em ambientes subtropicais, como relatado por Budke et al. (2005) em Floresta Estacional Decidual no Rio Grande do Sul. Este estudo indicou que esse padrão também ocorre na Floresta Ombrófila Mista. No entanto, por se tratar de floresta que passou por perturbações de corte seletivo no passado, a proporção de espécies que possuem zoocoria é alta (80,5\%), uma vez que, segundo Carvalho (2010), em florestas secundárias a estratégia de dispersão por animais é utilizada por $63,7 \%$ a $72,9 \%$ das espécies, enquanto somente em florestas preservadas esse número varia de $82,3 \%$ a $89,9 \%$.

A grande proporção de espécies zoocóricas confirma a importância dos animais para a dispersão de propágulos e, consequentemente, para a manutenção do fluxo gênico das florestas, de forma que a diminuição das populações de animais pode ter implicações diretas na eliminação ou diminuição de espécies zoocóricas (ALMEIDA et al., 2008). Espécies zoocóricas são também importantes para o plantio visando à recuperação de áreas degradadas, uma vez que atraem animais para essas áreas e a presença deles pode aumentar a velocidade da sucessão florestal. O fato de todas as espécies de Myrtaceae encontradas serem zoocóricas ressalta a importância dessa família como fonte de alimento para a fauna silvestre e o seu potencial de uso em plantios de recuperação de áreas degradadas.

As espécies autocóricas, todas da família Euphorbiaceae, confirmam a tendência à autocoria da família, padrão já observado em outros estudos, como de Nascimento et al. (2001), Rondon Neto et al. (2001), Budke et al. (2005) e Almeida et al. (2008).

A predominância de espécies com distribuição espacial aleatória contrasta com o resultado encontrado por Nascimento et al. (2001), que, estudando um fragmento de Floresta Ombrófila Mista em Nova Prata, RS, registraram a predominância de espécies com distribuição espacial agregada. As diferenças entre os dois estudos podem estar relacionadas a variações ambientais, a diferenças florísticas e ao histórico de perturbação das áreas.

Nas espécies com síndrome de dispersão zoocórica, a maior proporção de espécies também com distribuição espacial aleatória pode estar relacionada à dispersão a maiores distâncias feita por animais, de forma aleatória. A zoocoria exige da espécie vegetal alto gasto energético na produção de frutos, normalmente carnosos. No subbosque de matas densas, onde o vento não consegue realizar com eficiência a dispersão, a zoocoria é a melhor estratégia, pois reduz a competição entre as plântulas e a planta-mãe e também a predação de sementes e de plântulas próximas à árvore matriz (teoria da fuga, de JANZEN, 1970; CONNELL, 1971), aumentando, assim, a possibilidade de sucesso no recrutamento das espécies que, muitas vezes, são dispersas de forma aleatória. Além disso, de acordo com Budke et al. (2005), mesmo que o fruto caia da árvore-mãe, se possuir atrativos, ele pode ser disperso por agentes secundários, como formigas. No entanto, a distribuição de espécies zoocóricas seguindo um padrão aleatório contrasta com os resultados encontrados por Harms et al. (2000), Jordano e Godoy (2002) e Giehl et al. (2007), que encontraram espécies zoocóricas com predominância de distribuição agregada, pois os diásporos seriam depositados em lugares específicos, onde os animais permanecem por mais tempo. Variações no sistema de amostragem, no nível de inclusão dos indivíduos avaliados, no histórico de perturbação, entre outros, podem explicar as diferenças encontradas entre os estudos.

Quanto às espécies autocóricas, o fato de a maior proporção de espécies com essa síndrome se distribuir de forma agregada concorda com estudos realizados por Nascimento et al. (2001), Rondon Neto et al. (2001)e Almeida et al. (2008). Isso pode estar ocorrendo porque, normalmente, a autocoria limita a distribuição de propágulos para próximo da planta-mãe, pois, de acordo com Willson (1993), o alcance de dispersão por autocoria é pequeno. 
O fato de as espécies anemocóricas atingirem alturas mais elevadas é uma tendência esperada, pois, de acordo com Giehl et al. (2007), isso permite que o propágulo permaneça por mais tempo no ar, sujeito às diferentes velocidades e intensidades de ventos, além de existirem menos obstáculos aos diásporos, já que estes ficam acima do dossel. Contrariando esse padrão, encontra-se a única pteridófita arborescente amostrada na área, Dicksonia sellowiana Hook., que apresenta esporos dispersos pelo vento, altura mediana, e é uma espécie clímax de ocorrência em ambientes úmidos de sub-bosque.

Outra estratégia de espécies anemocóricas para diminuir os obstáculos na dispersão pelo vento é a perda das folhas durante a época de reprodução. De acordo com Leyser et al. (2009), em florestas estacionais, onde predomina a anemocoria, a perda das folhas em determinadas épocas do ano é comum, em virtude de garantir o sucesso na propagação. Apesar de a floresta estudada não ser floresta estacional, das 12 espécies anemocóricas, oito são caducifólias ou semicaducifólias (e.g. Clethra scabra Pers.) e apenas quatro são perenifólias.

Em relação às espécies autocóricas, a maior parte ocupou o estrato superior da floresta na área estudada. Tal fato corrobora Hughes et al. (1994), que afirmaram que, para evitar a competição intraespecífica, a plantamãe deve possuir maior tamanho em relação às demais presentes na comunidade, a fim de que seus diásporos sejam dispersados em maiores distâncias. Entretanto, devido ao fato de poucas espécies terem apresentado essa síndrome neste estudo, não é possível fazer inferências seguras sobre o comportamento de espécies autocóricas.

\section{CONCLUSÕES}

Neste estudo foi encontrada como forma de dispersão predominante a zoocoria, indicando estreita e importante relação entre a fauna e a flora silvestre. A maior proporção de espécies zoocóricas, seguidas por espécies anemocóricas, corrobora o padrão observado em outros trabalhos, tanto em florestas tropicais quanto em subtropicais.

Na distribuição espacial das populações, observouse predominância de espécies com padrão aleatório, tanto em relação ao todo quanto somente com relação às espécies zoocóricas. As espécies autocóricas, apesar de estarem representadas por poucos exemplares, apresentaram tendência à distribuição agregada, enquanto as anemocóricas não revelaram a predominância de um padrão espacial, ocorrendo tanto de forma agregada quanto aleatória.

A estratificação vertical da floresta demonstrou a predominância de espécies no estrato superior, seguida pelo estrato intermediário. Ao analisar a distribuição em alturas com as estratégias de dispersão, observase que a maior parte das espécies zoocóricas, anemocóricas e autocóricas também ocorre no estrato superior.

Conhecer essas estratégias é um ponto-chave no desenvolvimento de programas de manejo sustentável e de conservação de remanescentes florestais, importantes devido às inúmeras funções ecológicas que desempenham. Esse estudo também pode subsidiar projetos em que se deseja a atração da fauna nativa, como aqueles relacionados à recuperação de áreas degradadas e de arborização urbana, uma vez que se podem extrair da flora nativa espécies com potencial de uso para esses fins, como é o caso das espécies da família Myrtaceae, as quais possuem elevado número de interações com a fauna nativa.

\section{REFERÊNCIAS}

ALMEIDA, S. R. et al. F. Florística e síndromes de dispersão de um remanescente de Floresta Ombrófila Mista em sistema faxinal. Ambiência, v.4, n.2, p.289-297, 2008.

APG III. An update of the Angiosperm Phylogeny Group classification for the orders and families of flowering plants: APG III. Botanical Journal of the Linnean Society, v.161, n.2, p.105$121,2009$.

AUGSPURGER, C. K. Light requirements of neotropical tree seedlings a comparative study of growth and survival. Journal of Ecology, v.72, p.777-795, 1984.

BLATE, G. M.; PEART, D. R.; LEIGHTON, M. Post-dispersal predation on isolated seeds: a comparative study of 40 tree species in a Southeast Asian rainforest. Oikos, v.82, p.522-538, 1998.

Revista Árvore, Viçosa-MG, v.36, n.5, p.919-929, 2012 
BRASIL. Ministério da Agricultura e Reforma Agrária. Normais climatológicas 19611990. Brasília: 1992.84 p.

BUDKE, J. C. et al. Composição florística e estratégias de dispersão de espécies lenhosas em uma floresta ribeirinha, arroio Passo das Tropas, Santa Maria, RS, Brasil. Iheringia, Série Botânica, v.60, n.1, p.17-24, 2005.

CARVALHO, F. A. Síndromes de dispersão de espécies arbóreas de Florestas Ombrófilas Submontanas do estado do Rio de Janeiro. Revista Árvore, v.34, n.6, p.1017-1023, 2010.

CONNELL, J. H. On the role of natural enemies in prevent competitive exclusion in some marine animal and rainforest trees. In: DEN BOER, P. J.; GRADWELL, G. R. Dynamics of population proceedings of advanced study institute on dynamics of numbers in populations. Wageningen: Centre of Agricultural Publishing and Documentation, 1971. p.298-312.

ESKUCHE, U. El bosque de Araucaria con Podocarpus y los campos de Bom Jardim da Serra, Santa Catarina (Brasil meridional). Boletín de la Sociedad Argentina de Botánica, v.42, n.3-4, p.295-308, 2007.

FALKENBERG, D. Matinhas nebulares e vegetação rupícola dos Aparados da Serra Geral (SC/RS), sul do Brasil. 2003. 558f. Tese (Doutorado em Biologia Vegetal) - Universidade Estadual de Campinas, Campinas, 2003.

FORMENTO, S. et al. Dinâmica estrutural arbórea de uma Floresta Ombrófila Mista em Campo Belo do Sul, SC. Cerne, v.10, n.2, p.196-212, 2004.

GIEHL, E. L. H. et al. Espectro e distribuição vertical das estratégias de dispersão de diásporos do componente arbóreo em uma floresta estacional no sul do Brasil. Acta Botanica Brasílica, v.21, n.1, p.137-145, 2007.

GOTTSBERGER, G; SILBERBAUERGOTTSBERGER, I. Dispersal and distribution in the cerrado vegetation of Brazil. Sonderbänd des Naturwissenschaftlichen Vereins in Hamburg, v. 7, p.315-352, 1983.

Revista Árvore, Viçosa-MG, v.36, n.5, p.919-929, 2012
HARMS, K. E. et al. Pervasive densitydependent recruitment enhances seedling diversity in a tropical forest. Nature, v.404, p.493-495, 2000.

HIGUCHI, P. et al. Influência de variáveis ambientais sobre o padrão estrutural e florístico do componente arbóreo em um fragmento de Floresta Ombrófila Mista Montana, em Lages, SC. Ciência Florestal, v.12, n.1, p.79-90, 2012.

HIGUCHI, P. et al. Florística e estrutura do componente arbóreo e análise ambiental de um fragmento de Floresta Ombrófila Mista Altomontana no município de Painel, SC. Ciência Florestal, no prelo.

HUBBELL, S. P. Seed predation and coexistence of tree species in tropical forests. Oikos, v.35, n.2, p.214-229, 1980.

HUGHES, L. et al. Predicting dispersal spectra: a minimal set of hypotheses based on plant attributes. Journal of Ecology, v.82, n.4, p.933-950, 1994.

\section{INSTITUTO BRASILEIRO DE GEOGRAFIA E} ESTATÍSTICA - IBGE. Manual técnico da vegetação brasileira. Rio de Janeiro: 1992. 92 p. (Série: Manuais Técnicos em Geociências, 1)

JANZEN, D. H. Herbivores and the number of tree species in tropical forests. American

Naturalist, v.104, n.940, p.501-528. 1970.

JORDANO, P.; GODOY, J. A. Frugivore-generated seed shadows: a landscape view of demographic and genetic effects. In: LEVEY, D. J.; SILVA, W. R.; GALleti, M. Seed dispersal and frugivory: ecology, evolution and conservation. New York: CABI Publishing, 2002. p.305-321.

LEYSER, G. et al. Espectro de dispersão em um fragmento de transição entre Floresta Ombrófila Mista e Floresta Estacional na região do Alto Uruguai, Rio Grande do Sul, Brasil. Pesquisas, série botânica, v.60, p.355-366, 2009.

LORENZI, H. Árvores brasileiras: manual de identificação e cultivo de plantas arbóreas nativas do Brasil. 2.ed. Nova Odessa: Plantarum, 1998a. v.1. 
LORENZI, H. Árvores brasileiras: manual de identificação e cultivo de plantas arbóreas nativas do Brasil. 2.ed. Nova Odessa: Plantarum, 1998b. v.2.

LORENZI, H. Árvores brasileiras: manual de identificação e cultivo de plantas arbóreas nativas do Brasil. 2.ed. Nova Odessa: Instituto Plantarum, 2009. v.3.

MORISITA, M. Id-index, a measure of dispersion of individuals. Researches on population ecology, v.4, p.1-7, 1962.

NASCIMENTO, A. R. T.; LONGHI, S. J.; BRENA, D. A. Estrutura e padrões de distribuição espacial de espécies arbóreas em uma amostra de Floresta Ombrófila Mista em Nova Prata, RS. Ciência Florestal, v.11, n.1, p.105-119, 2001.

NUNES, Y. R. F. et al. Variações da fisionomia, diversidade e composição de guildas da comunidade arbórea em um fragmento de floresta semidecidual em Lavras, MG. Acta Botanica Brasílica, v.17, n.2, p.213-229, 2003.

PERES, C. A.; SCHIESARI, L. C.; DIAS-LEME, C. L. Vertebrate predation of Brazil-nuts (Bertholetia excelsa, Lecythidaceae), an agouti-dispersed Amazonian seed crop: a test of the escape hypothesis. Journal of Tropical Ecology, v.13, n.1, p.69-79, 1997.

RONDON NETO, R. M.; WATZLAWICK, L. F.; CALDEIRA, M. V. W. Diversidade florística e síndromes de dispersão de diásporos das espécies arbóreas de um fragmento de Floresta Ombrófila Mista. Revista Ciências Exatas e Naturais, v.3, n.2, p.167-175, 2001.
SMITH-GILL, S. J. Cytophysiological basis of disruptive pigmentary patterns in the leopard frog, Rana pipiens. II. Wild type and mutant cell specific patterns. Journal of Morphology, v.146, n.1, p.35-54, 1975.

SILVA, A. C. et al. Relações florísticas e fitossociologia de uma Floresta Ombrófila Mista Montana secundária em Lages, Santa Catarina. Ciência Florestal, v.12, n.1, p.193-206, 2012.

SOUZA, D.; SOUZA, A.; LEITE, H. Emprego de análise multivariada para estratificação vertical de florestas ineqüiâneas. Revista Árvore, v.27, n.1, p.59-63, 2003.

URBANETZ, C.; OLIVEIRA, V. M.; RAIMUNDO, R. L. G. Padrão espacial, escala e síndromes de dispersão, 2003. Disponível em: <http://www2.ib.unicamp.br/profs/fsantos/ relatorios/ne211r3a2003.pdf $>$ Acesso em: 10 mar. de 2011 .

van der PIJL, L. Principles of dispersal in higher plants. Berlin: Springer-Verlag, 1982. 162p.

WILLSON, M. F. Dispersal mode, seed shadows, and colonization patterns. Vegetatio, v.107-108, NUMERO, p.261-280, 1993.

WRIGHT, S. J.; DUBER, H. C. Poachers and forest fragmentation alter seed dispersal, seed survival, and seedling recruitment in the palm Attalea butyracea, with implications for tropical tree diversity. Biotropica, v.33, n.4, p.583-595, 2001.

ZIPARRO, V. B.; MORELLATO, L. P. C. Seed predation of Virola bicuhyba (Schott) Warb. (Myristicaceae) in the Atlantic forest of southeastern Brazil. Revista Brasileira de Botânica, v.28, n.3, p.515-522, 2005. 
